\title{
Investigation and Analysis in the Case of Belgian Learners' Use of Comparative Sentences in Written Production
}

\author{
Hongjuan Liu \\ International College of Qujing Normal University, Yunnan, Chin \\ 763678246@qq.com
}

\begin{abstract}
Making errors is an inevitable and necessary part of learning a second language. It will be very helpful for Chinese teachers to know what errors the learners will make and also the sources of these errors if they can make full use of the results of error analysis. Therefore, we can grasp the initiative to eliminate the errors in the bud effectively. The purpose of the current study is to find Belgian learners' acquisition rules of Chinese Comparative Sentences by investigating the case of their use of comparative sentences in written production, in order to get a definite aim in the teaching process to reduce or avoid the occurrence of such errors effectively. So as to make the students' interlanguage system get close to the target language system as soon as possible.
\end{abstract}

Keywords: Error; Comparative sentence; Belgian students; Investigation

\section{比利时学生书面表达中使用汉语比较句情况的调}

\section{查和分析}

刘红娟

（曲靖师范学院国际学院，云南省曲靖市 655011 )

摘要: 偏误的出现是第二语言学习过程中的正常现象, 对学习者目的语系统的形成不仅是无法避免的, 而且也是十分必 要的。充分利用偏误分析的结果, 利于教师预先了解学习者可能产生的偏误及偏误的来源, 这样在教学中能够掌握主动, 有 效将偏误消灭于萌芽状态 ${ }^{[1]}$ 。通过对处于中介语阶段的比利时学习者汉语写作材料中出现的汉语比较句偏误情况的调查, 发 现他们对汉语比较向的习得规律, 从而在教学过程中有的放矢, 有效地减少或避免类似偏误的出现, 利于使学生的中介语系 统尽快地向目的语系统靠拢。

关键词：偏误; 比较句;比利时学生;调查

\section{引言}

偏误分析在第二语言习得研究中的重要性最早由科德在 1967 年提出 [1], 之后该领域有大量关于偏误 研究的成果, 但大部分的研究都集中于拼音文字, 很少有人关注留学生将汉语作为第二语言来学习时所产 生的偏误。国内关于偏误分析的研究始于鲁健瞕先生发表的《中介语理论与外国人学习汉语的语音偏误分 析》一文[2], 之后对外汉语教学界虽然发表了大量涉及汉语语音、词汇、语法、汉字、语用、语篇等的 偏误分析的文章, 但大部分的思路还局限于采用描述性研究及解释性研究两种方法, 实证性的研究很少。 以留学生学习汉语比较句的偏误为例, 我们以 “偏误分析” “比较句” “对外汉语” 三个关键词的组合为依 据搜索了中国学术期刊网从 1994 年至今的全部期刊, 符合搜索条件的文章高达 200 多篇, 然而这其中不 少文章完全是从本体研究的角度对汉语比较句进行描述与分析的, 还有一部分是将汉语比较句与学生的母 语中相关的句型进行对比分析的, 这种研究的价值在于从语言学的角度让我们对汉语比较句的特点有了更 加清楚的认识, 而从第二语言教学的角度而言, 实际应用价值有限。其中真正符合 “从第二语言学习的角 
度来研究汉语比较句的” “相关研究中使用了偏误分析的方法” “文中偏误语料均来源于对留学生学习汉语 比较句实际情况的调查”这三个条件的文章只有 5 篇 [3] [4] [5] [6] [7]。

以上文章的研究结果显示, 来自不同母语背景的学生在习得汉语比较句的过程中, 其偏误的类型既有 差异性, 也存在着许多共性。同时这些偏误语料绝大多数来源于韩国、日本及东南亚国家的学生, 欧美学 生所占比例很少。因此研究比利时学生学习汉语比较句的偏误。一方面弥补了以前从未有人专门以比利时 学生为调查对象进行相关研究的不足; 另一方面利用偏误分析的研究成果, 有利于我们提前预知比利时学 生在学习汉语比较句时常见的偏误及偏误出现的原因, 利于教师提前采取有效措施帮学生减少或避免类似 偏误的出现, 提高教学效率。

\section{1 调查的目的}

美国语言教育学家塞林克 (L. Selinker) 于 1969 年提出了中介语（interlanguage）的概念, 所谓 “中 介语” 是指在第二语言的习得过程中, 学习者通过一定的学习策略, 在目的语输入的基础上所形成的一种 既不同于其第一语言, 也不同于目的语, 随着学习的进展向目的语逐渐过渡的动态的语言系统, 它反映了 学习者从零起点到靠近目的语的语言发展轨迹 [8]。对中介语的研究有利于探索学习者语言系统的本质, 发现第二语言习得的发展阶段，揭示第二语言的习得过程及第一语言的影响。

本文试图通过对处于中介语阶段的比利时学习者汉语写作材料中出现的汉语比较句偏误情况的调查, 发现他们对汉语比较句的习得规律, 从而在教学过程中有的放矢, 有效地减少或避免类似偏误的出现, 使 学生的中介语系统尽快地向目的语系统靠拢。

\section{2 调查的对象、方法及内容}

考虑到不同水平的学生对汉语比较句的习得程度不同, 本文的调查对象是比利时荷语鲁汶大学本科二 年级及三年级的学生, 目前三年级的学生就读一、二年级时的综合课教师, 与现在二年级学生一二年级时 的综合课教师是一样的, 因此就课堂学习而言, 他们最初所接受的关于比较句的教育是基本一致的。之所 以选择这两个年级的学生作为调查对象, 一方面通过对这两个年级的学生生成的比较句偏误共性的比较, 有利于发现一二年级综合课教学中关于比较句的教学所存在的问题; 另一方面, 通过对他们所生成的不同 偏误的分析, 也能让我们更好地了解他们对汉语比较句的习得规律。

本次调查以书面调查为主, 采用限时写作及不限时写作两种形式。限时写作的时间为 35-40 分钟, 两 个学生一起合作, 根据教师给出的比较结构, 就某两种事物、两种文化或者两种现象等进行比较, 二三年 级教师所给的比较结构是一样的。这样虽然有一定的人为诱导成分, 但它能够较为集中地考察留学生对汉 语比较句的习得情况。不限时写作是学生的一次正常的课后习作, 要求学生就某两种事物、两种文化或者 两种现象等进行比较, 教师未给出相应的比较结构。之所以选择书面写作的调查方式, 一方面是因为此时 的偏误是在留学生有较充分的思考时间的前提下产生的, 因此它们能够较为真实地反映留学生对汉语比较 句的实际习得水平; 另一方面, 书面写作中出现的句子不是孤立的, 它需要符合上下文语境的要求, 更利 于我们发现学生在使用汉语比较句时在语用方面出现的偏误。

\section{3 比较句范围的确定及偏误确定的标准}

本文所选的比较句限于学生作文中出现的有明显标志词的句子, 包括 “X 跟 Y 一样 (不一样、差不多 
等）+W” (1) “X 没有 $Y+W$ “ “ $X$ 不如 $Y+W$ “ “ $X$ 不比 $Y+W$ ” “X 比 $Y+W$ ” “更/最 $+A d j / V$ ” “越来越 $+A d j / V$ ” 及 “跟 $X$ 比起来, $Y \cdots \cdot . .$. ” 这样的几个句式, 至于文中出现的表示比较, 但却无明显标志词的表达方式, 如 “中国 人的薪水每个月平均到 584 欧元, 这只是比利时人薪水的 $30 \%$ ” “荷兰人的性格粗蓛, 不会拐弯抹角, 很外 向。比利时人很内向。”等不列为本文所选比较句的范畴。

英国应用语言学家科德 (Corder) 把学习者所犯的错误分为失误 (mistake) 和偏误 (errror) 两种。 失误是指偶然产生的口误或笔误, 它没有什么规律, 说话者一旦意识到马上可以自己改正, 并且以后不一 定再出现这类错误。偏误则是指由于对目的语掌握得不好而产生的一种规律性错误, 它偏离了目的语的轨 道, 反映了说话者的语言能力和水准 [1]。本文同意科德的偏误确定标准, 在所收集的语料中, 我们只关 心与汉语比较句的句法、语义、语用相关的偏误, 与此无关的其他偏误, 包括学生对相关词语的选用不当, 如 “林堡的自然 (根据文本揣测, 他要表达的是 “植被”) 比安特卫普的多。”, “狄老师跟黎老师一样滑㹈。 (学生可能要说 “幽默”)”, 学生所写的错别字, 如 “狄老师比黎老师年齿 (应为 “龄”)” 及其他与比较 句本身不相关的表达偏误，如 “男人不如女人那么好看得到别人有问题的话。”（实际应为 “容易发现别人 说的话不对劲”) 等, 我们都不记为偏误。此外, 有些句子可能完全符合句法规则, 但放在一定的语境中 则不符合语用要求, 如一个学生在文中提到结婚以后就不会孤独了, 但之后却写了 “*单身的生活比结婚 的生活更孤独” (2)这样的句子, 我们将其视为偏误。同时同一个学生出现的完全相同的偏误, 我们只记做 一次, 如一个学生在同一篇文章里出现了 “单身比结婚的人自由得多。” “结婚的人不如单身有钱。” “单身 比结婚的人孤独一点儿” 这样几个带有偏误的句子, 其偏误的原因都在比较项 “单身” 与 “结婚的人” 语 义上不具可比性造成的, 因此其偏误数我们只记做一个。

\section{4 调查结果的分析}

表 1 限时写作中二三年级学生出现的偏误情况比较

\begin{tabular}{|c|c|c|c|c|c|c|}
\hline \multirow{2}{*}{ 句型 } & \multicolumn{5}{|c|}{ 年级 } \\
\cline { 2 - 7 } & \multicolumn{3}{|c|}{ 二年级 } & \multicolumn{3}{c|}{ 三年级 } \\
\cline { 2 - 7 } & 偏误数 & 总数 & 偏误率 & 偏误数 & 总数 & 偏误率 \\
\hline $\mathrm{X}$ 跟 $\mathrm{Y}$ 一样+W & 3 & 20 & $15 \%$ & 0 & 10 & 0 \\
\hline $\mathrm{X}$ 比 $\mathrm{Y}+\mathrm{W}$ & 21 & 61 & $34.4 \%$ & 6 & 27 & $22.2 \%$ \\
\hline $\mathrm{X}$ 没有 $\mathrm{Y}+\mathrm{W}$ & 0 & 5 & 0 & 2 & 9 & $22.2 \%$ \\
\hline $\mathrm{X}$ 不如 $\mathrm{Y}+\mathrm{W}$ & 0 & 6 & 0 & 1 & 11 & $9.1 \%$ \\
\hline $\mathrm{X}$ 不比 $\mathrm{Y}+\mathrm{W}$ & 0 & 0 & 0 & 4 & 4 & $100 \%$ \\
\hline 更/最 $+\mathrm{Adj} / \mathrm{V} ”$ & 0 & 1 & 0 & 0 & 0 & 0 \\
\hline 越来越 $+\mathrm{Adj} / \mathrm{V}$ & 0 & 0 & 0 & 0 & 0 & 0 \\
\hline 跟 X 比起来, $\mathrm{Y} \cdots \cdots$ & 0 & 0 & 0 & 0 & 0 & 0 \\
\hline 总数 & 24 & 93 & $25.8 \%$ & 13 & 61 & $21.3 \%$ \\
\hline
\end{tabular}

(1) 本文中出现的 X 均指比较前项, $Y$ 指比较后项, W 指比较结果。

(2) “*”表示这个句子带有偏误的向子, 下文同。 
我们收集到的限时写作共计 25 篇, 其中二年级的 13 篇, 三年级的 12 篇, 两个年级学生的写作数量 大致相当。表 1 中的数据显示二年级的学生选用带有标志性词语的比较句式共生成句子 93 个, 三年级的 学生生成的相关句子是 61 个, 明显少于二年级的。这一方面可能与二年级学生才刚刚集中学完表示比较 的相关句式, 因此对其比较熟悉, 也乐于加以运用相关: 另一方面, 随着汉语水平的提高, 三年级的学生 可以更多的选择其他句式对某两种事物或现象进行比较, 因此他们可能对带有标志性词语的比较句式采用 了一定的回避策略。这也提醒我们的对外汉语教师, 在实际教学过程中不仅要注意所教词汇的复现, 也要 注重对相关语法点的复现。语法点的适当复现, 不仅有助于帮助学生对所学知识的复习与巩固, 鼓励他们 用相关的句式进行语言交际, 同时也能很好地补充之前因学生汉语水平所限, 教师未能讲出或未能充分讲 清的一些知识点。正如吕文华先生所提倡的要注重语法教学的分级, 这种语法分级既包括将语法系统横向 地划分为初级、中级、高级三个学习阶段, 也包括将同一语法项目作纵向的层次划分 [9]。

通过表 1 我们还可以看到二年级学生所选的带有标志性词语的比较句式比较集中在 “X 跟 $Y$ 一样+W” 及 “X 比 $\mathrm{Y}+\mathrm{W}$ ” 上, 这两个句式是学生最先接触的两个比较句式, 因而他们更熟悉。与三年级学生相比, 二年级学生在运用这两个句式时产生的偏误率明显高于三年级学生的偏误率, 这也表明在教师已教授完比 较句式的前提下, 学生可以在实际运用过程中自觉或不自觉地改正自己在汉语习得方面的一些偏误, 实现 中介语向目的语的不断靠拢, 因此随着三年级学生汉语水平的提高, 其对汉语比较句的习得情况越来越好。 但是通过观察我们也可以发现三年级学生在总的偏误率上只比二年级的学生低一点儿, 这一方面可能因为 随着学生汉语词汇量的增加, 他们要表达的内容越来越复杂, 因而原有的简单句式满足不了他们的表达要 求, 使用复杂句式时出现偏误的机率当然更高, 比如三年级的学生选用了二年级的学生所未用的 “ $X$ 不比 $\mathrm{Y}+\mathrm{W}$ ” 这样的句式, 在 “X 不如 $\mathrm{Y}+\mathrm{W}$ ” 及 “X 没有 $\mathrm{Y}+\mathrm{W}$ ” 这两个句式的选用上, 三年级学生生成的句子也明 显多于二年级的。另一方面这也符合偏误具有反复性的特点, 即留学生在开始学习汉语比较句式时已经被 纠正了的偏误在后期又可能重现了。这就提醒我们对外汉语教师要注意语法教学的层次性, 如在教师已明 确给出 “ $X$ 不比 $Y+W ” ~ “ X ~$ 不如 $Y+W ”$ 及 “ $X$ 没有 $Y+W ”$ 这三个句式的前提下，二年级学生运用这三个句式生 成的句子只占全部比较句的 $11.8 \%$, 且没有任何一个学生选用 “ $X$ 不比 $Y+W ”$ 这个句式, 我们是否可以考虑 延迟 “X 不比 $\mathrm{Y}+\mathrm{W}$ ” 这个句式的教学, 将它放在三年级, 而非根据现有教材的安排将所有的比较句式给学 生集中在一段时间内讲完。关于 “ $X$ 不如 $Y+W$ ” “ “ $X$ 没有 $Y+W$ ” 这两个句式, 通过搜集二年级学生生成的 句子，我们发现只限于简单的诸如 “鲁汶的男生没有安特卫普的男生那么帅” “比利时的运动员没有中国 的运动员那么多” 这样的句子, 因此在二年级阶段教师可以简单的介绍这两个句式中 W 是形容词, X、Y 分 别为名词、代词或体词性结构的情况, 而对于 W 为谓词性结构, 如 “我没有他能喝酒。” “我不如他跑得 快。”，使用这两个句式在语义语用方面的限制及这两个句式之间的联系与区别则可以推迟到三年级去讲， 这样既避免了语法的过度集中, 也保证了比较句式这一语法点的适度重现, 可以有效防止留学生在开始学 习汉语比较句式时已经被纠正了的偏误在后期又重现。 
表 2 限时写作与非限时写作中三年级学生出现的偏误情况比较

\begin{tabular}{|c|c|c|c|c|c|c|}
\hline \multirow{3}{*}{ 句型 } & \multicolumn{6}{|c|}{ 类别 } \\
\hline & \multicolumn{3}{|c|}{ 非限时 ${ }^{(1)}$} & \multicolumn{3}{|c|}{ 限时 } \\
\hline & 偏误数 & 总数 & 偏误率 & 偏误数 & 总数 & 偏误率 \\
\hline $\mathrm{X}$ 跟 Y 一样+W & 5 & 37 & $13.5 \%$ & 0 & 10 & 0 \\
\hline $\mathrm{X}$ 比 $\mathrm{Y}+\mathrm{W}$ & 10 & 37 & $27 \%$ & 6 & 27 & $22.2 \%$ \\
\hline $\mathrm{X}$ 没有 $\mathrm{Y}+\mathrm{W}$ & 6 & 14 & $42.9 \%$ & 2 & 9 & $22.2 \%$ \\
\hline X 不如 Y+W & 0 & 3 & 0 & 1 & 11 & $9.1 \%$ \\
\hline X 不比 Y+W & 1 & 2 & $50 \%$ & 4 & 4 & $100 \%$ \\
\hline 更/最+Adj/V” & 4 & 12 & $33.3 \%$ & 0 & 0 & 0 \\
\hline 越来越+Ad j/V & 0 & 1 & 0 & 0 & 0 & 0 \\
\hline 跟 X 比起来, $Y \cdots \cdots \cdot$. & 0 & 2 & 0 & 0 & 0 & 0 \\
\hline 总数 & 26 & 108 & $24.1 \%$ & 13 & 61 & $21.3 \%$ \\
\hline
\end{tabular}

表 2 显示，无论是学生小组合作限时完成的句子，还是学生在有充分考虑时间的前提下个人完成的句 子, 其运用比较句进行表达时出现的偏误情况比较一致, 这也反映了学生对这些表达句式的实际习得水平。 学生使用最多的句式是 “ $X$ 跟 $Y$ 一样+W” 及 “ $X$ 比 $Y+W ”$, 其中 “ $X$ 跟 $Y$ 一样+W” 这一句式学生习得情况比 较好。偏误率最高的两个句式是 “ $\mathrm{X}$ 不比 $\mathrm{Y}+\mathrm{W}$ ” 和 “ $\mathrm{X}$ 没有 $\mathrm{Y}+\mathrm{W}$ ”, 这也再次提醒我们, 直到三年级学生还 未能很好地掌握的句式，不宜在二年级一股脑全部教给学生，那样势必事倍功半。

表 3 二三年级学生出现的偏误类型的比较

\begin{tabular}{|c|c|c|c|c|c|c|c|c|}
\hline \multirow{3}{*}{ 年级 } & \multicolumn{8}{|c|}{ 偏误类型 } \\
\hline & \multicolumn{2}{|c|}{ 比较项 } & \multicolumn{2}{|c|}{ 比较结果 } & \multicolumn{2}{|c|}{$\begin{array}{r}\text { 句式混淆 } \\
\text { 或杂糅 }\end{array}$} & \multicolumn{2}{|c|}{ 其他 } \\
\hline & 偏误数 & 偏误率 & 偏误数 & 偏误率 & 偏误数 & 偏误率 & 偏误数 & 偏误率 \\
\hline 二年级 & 7 & $29.2 \%$ & 8 & $33.3 \%$ & 6 & $25 \%$ & 3 & $12.5 \%$ \\
\hline 三年级 & 20 & $51.3 \%$ & 7 & $17.9 \%$ & 7 & $17.9 \%$ & 5 & $12.8 \%$ \\
\hline
\end{tabular}

表 3 显示无论二年级还是三年级的学生在比较项及比较结果两方面出现的偏误都超过了偏误数的一半 以上，其中绝大部分涉及到相关句式的句法结构使用不当，一部分是与其结构成分的语义相关; 句式混淆 或杂糅则多是语用选择不当。我们尤其注意到三年级的学生比较项的偏误占了全部偏误数的一半以上, 这 一方面与其所表达的内容越来越复杂，因而出现偏误的几率高有关系; 另一方面也表明在比较句式为非正 在学习的重要语法点的前提下, 学生对相关语法点的句法结构重视程度降低, 片面追求交际的可理解性, (1) 非限时写作收到习作 25 篇，限时写作是小组合作的形式，收到习作 12 篇。 
而忽视了语法点使用的准确性。通过分析三年级学生生成的比较项带有偏误的比较句, 我们发现很大一部 分偏误都是因将两个语义不对等的比较项进行比较, 或者当 $X$ 和 $Y$ 为具有相同中心语的偏正结构时, 中心 语成分的省略不当造成的，如 “*夫妇比单身还快乐。” “*中国的消费价格比比利时低 $44 \%$ ” “*比利时的产 品质量常常比荷兰产品好” “*比利时人的生活比中国的轻松得很” 等, 而这两点应该是教师在讲解相关 比较句式时重点强调的内容, 学生经过一段时间的消化、运用之后, 不仅没有学得更好, 反而退步了, 因 此我们必须强调语法教学应贯穿于留学生汉语学习的各个阶段, 而不能全部集中于初级阶段。

\section{5 结论}

比利时王国虽然面积不大, 人口也不多。但由于特殊的政治、地理及宗教等原因, 这个国家拥有法语 和荷兰语两种种官方语言, 此外德语和英语在一定的地区也非常通行, 还有一些移民讲阿拉伯语、土耳其 语、意大利语、葡萄牙语、东欧语言等。在这种多语的环境中, 不少比利时的孩子从小就接受双语甚至是 三语或多语教育, 因此可以说大部分比利时人都是语言天才, 擅长讲多种语言 [10]。丰富的外语学习经验 对他们的汉语学习起到了良好的促进作用, 因此相对于处于单语环境中的学习者而言, 比利时学生在汉语 学习方面具有得天独厚的优势, 因此如果我们的汉语教师在教学过程中能够充分考虑比利时学生自身的学 习特点, 并根据偏误调查的结果提前预知比较句教学的重点与难点, 同时参考汉语教学的客观规律, 科学、 合理的安排教学内容, 必然会使得教学效果事半功倍。

\section{6 致谢}

曲靖师范学院校级课题《比利时学生学习汉语比较句的偏误分析》 (编号: 2015 QN006)

Research Project of Qujing Normal University named 'Error analysis of Belgian learners' use of comparative sentences" (No. 2015 QN006 ).

\section{参考文献:}

[1] Corder, S. P. The significance of learners' errors[J]. International Review of Applied Linguistics, 1967, No. 5. p. $161-170$.

[2] 鲁健冀. 中介语理论与外国人学习汉语的语音偏误分析 [J]. 语言教学与研究, 1983, 3:44一 56 .

[3] 钱 颖. 基于中介语语料库的比较句中程度副词的偏误分析一一以日韩学生为例 $[\mathrm{J}]$.. 华中人文 论丛, 2012, 6: 85-87.

[4] 宋 鹏. 柬埔寨留学生汉语比较句研究综述 [J]. 和田师范专科学校学报, 2011, 7:57-59.

[5] 陈 珺, 周小兵. 比较句语法项目的选取和排序 [J]. 语言教学与研究, 2005, 2:22-32.

[6] 刘红娟. 对外国留学生学习使用汉语比较句情况的调查和分析 [J]. 现代语文, 2009, 33: 135-137

[7] 肖小平. 越南留学生学习汉语比较句的结论想偏误分析 [J]. 海外华文教育, 2012, 2:184-190

[8] Selinker, L. Interlanguage[J]. International Review of Applied Linguistics, 1972, 10:209 $-231$.

[9] 吕文华. 对外汉语教学语法探索 [M]. 增订本. 北京: 北京语言大学出版社，2008:96

[10] 刘红娟. 比利时汉语教学概况 $[J]$. 云南师范大学学报 (对外汉语教学与研究版), 2012, 10 (6): 


\section{References}

[1] Corder, S. P: The significance of learners' errors [J].International Review of Applied Linguistics, 1967, 5: 161-170.

[2] Lu Jian-ji:A study on the theory of Interlanguage and the Phonetic error analysis of foreign Chinese learners[J].Language teaching and research,1983,No.3,p.44-56.(In Chinese)

[3] Qian Ying: Interlanguage corpus analysis of the use of adverbs in comparative sentences: A case study of Korean and Japanese students [J].Journal of Huazhong Normal University, 2012, No. 6, p.85-87.(In Chinese)

[4] Song Peng: Error analysis on Cambodian students' use of comparative sentences[J]. Journal of Hotan Teachers College, 2011, No. 7,p. 57-59. (In Chinese)

[5] Chen Jun. \& Zhou Xiaobing: The selection and arrangement of grammatical items concerning comparative sentences [J].Language Teaching and Linguistic Studies,2005, No.2,p.22-33.(In Chinese)

[6] Liu Hongjuan: Investigation of the use of comparative sentences by CFL learners[J]. Modern Chinese, 2009, No.11, p. 135-137. (In Chinese)

[7] Xiao Xiaoping: Analysis of Vietnamese Students'errors in the acquisition of the conclusion section in Chinese comparative sentences [J].Overseas Chinese Education, 2012, No.2,p184-190.(In Chinese)

[8] Selinker, L: Interlanguage[J].International Review of Applied Linguistics, 1972, No.10, p. 209-231.

[9] Lv Wenhua: Exploration of teaching Chinese as a foreign language[M].Beijing:Beijing Language and Culture University press,2008,p.96(In Chinese)

[10] Liu Hongjuan: A Summary Review of Chinese Teaching in Belgium[J].Journal of Yunan Normal University (Teaching and Research on Chinese As A Foreign LanguageEdition), 2012,No.6, p.84-89.(In Chinese) 